\title{
Classificação dos sistemas de produção e grau de impacto ambiental das pisciculturas no estado do Amazonas, Brasil
}

Silva Lima, Carlos André; Fragoso Machado-Bussons, Márcia Regina; Pantoja-Lima, Jackson

Classificação dos sistemas de produção e grau de impacto ambiental das pisciculturas no estado do Amazonas, Brasil

Revista Colombiana de Ciencia Animal, vol. 11, núm. 1, 2019

Universidad de Sucre, Colombia

DOI: https://doi.org/10.24188/recia.v11.n1.2019.707

Los autores permiten a RECIA reimprimir el material publicado en él. En caso de que un autor quiera traducir o usar una publicación parcial o completa de nuestro Diario, el autor debe obtener un permiso por escrito del editor de la revista.

Esta obra está bajo una Licencia Creative Commons Atribución 4.0 Internacional. 


\title{
Classificação dos sistemas de produção e grau de impacto ambiental das pisciculturas
} no estado do Amazonas, Brasil

\author{
Classification of production systems and degree of environmental impact of fishfarming in the state of Amazonas, \\ Brazil \\ Clasificación de los sistemas de producción y grado de impacto ambiental de las pisciculturas en el estado de \\ Amazonas, Brasil
}

Carlos André Silva Lima

Universidade Federal do Amazonas (UFAM). Av. General

DOI: https://doi.org/10.24188/recia.v11.n1.2019.707

Rodrigo Octávio, 6.200, Coroado - CEP: 69.077-000 -

Manaus - AM - Brasil, Brasil

helter_tgz@hotmail.com

iD http://orcid.org/0000-0003-3862-0873

Recepção: 10 Fevereiro 2019 Aprovação: 26 Abril 2019

Publicado: 06 Maio 2019

\author{
Márcia Regina Fragoso Machado-Bussons \\ Escola Superior Batista do Amazonas (ESBAM). Rua \\ Leonor Teles, 153 - Adrianópolis, Manaus - AM - CEP: \\ 69.057-510 - Manaus - AM - Brasil., Brasil \\ mmachadobussons@gmail.com \\ iD http://orcid.org/0000-0002-5038-1775
}

\section{Jackson Pantoja-Lima}

Instituto Federal de Educação do Amazonas (IFAM). Av.

Onça Pintada, 1.380, Galo da Serra - CEP: 69.735-000 -

Manaus - AM - Brasil., Brasil

jackson.lima@ifam.edu.br

iD http://orcid.org/0000-0002-6449-4981

\section{Resumo:}

A piscicultura é uma das atividades zootécnicas que mais tem crescido nos últimos anos, em especial na Amazônia Brasileira. O presente estudo foi realizado na área de abrangência do estado do Amazonas, um dos maiores consumidores de pescado no Brasil, tendo como objetivo caracterizar os sistemas de produção, o grau de severidade de impactos ambientais que essa atividade promove e as diferentes perspectivas de desenvolvimento futuro nas distintas mesorregiões do estado. As investigações foram realizadas por meio da coleta de dados secundários fornecidos pelo Instituto de Proteção Ambiental do Amazonas (IPAAM), que possui 1.382 pisciculturas registradas em seu banco de dados. Essas informações foram organizadas por microrregião (centro, sudoeste, norte e sul) do estado, explorando seus principais indicadores técnicos, as infraestruturas de produção de formas jovens, o tamanho dos empreendimentos e o enquadramento do grau de severidade de impacto ambiental por porte da piscicultura. Os resultados obtidos mostram que a piscicultura é praticada majoritariamente para engorda de tambaqui com finalidade de comercialização, realizada em estruturas de terra firme (viveiros escavados e barragens) em pequenas áreas alagadas, com aplicação de sistema semiintensivo de produção, sendo desenvolvida próximo a centro consumidor (Manaus), tendo como causa da sua concentração fatores logísticos e de infraestrutura disponíveis, com a maioria dos empreendimentos enquadrados como baixo grau de severidade de impacto ambiental.

Palavras-CHave: Colossoma macropomum, Amazônia, legislação ambiental, semi-intensivo, criação de peixes.

\section{Abstract:}


Fish farming is one of the zootechnical activities that has grown the most in recent years, especially in the Brazilian Amazon. The present study was carried out in the area of the State of Amazonas, one of the largest consumers of fish in Brazil, aiming at characterizing the production systems, the degree of severity of environmental impacts that this activity promotes and the different perspectives of future development in the different mesoregions of the state. The investigations were carried out through the collection of secondary data provided by the Institute of Environmental Protection of Amazonas (IPAAM), which has 1,382 fish farms registered in its database. This information was organized by the state's microregion (center, south-west, north and south), exploring its main technical indicators, production infrastructures of young forms, size of enterprises and the degree of severity of environmental impact by size of fish farming. The results obtained show that fish farming is practiced mainly for fattening of tambaqui for commercial purposes, carried out in terra firme structures (excavated nurseries and dams) in small flooded areas, with application of semi-intensive production system, being developed near (Manaus), due to its concentration of available logistical and infrastructure factors, with most of the projects classified as low severity of environmental impact.

KEYWORDS: Colossoma macropomum, Amazônia, environmental law, semi-intensive, fishfarming.

\section{Resumen:}

La piscicultura es una de las actividades zootécnicas que más ha crecido en los últimos años, en especial en la Amazonia brasileña. El presente estudio fue realizado en el área de cobertura del estado de Amazonas, uno de los mayores consumidores de pescado en Brasil, teniendo como objetivo caracterizar los sistemas de producción, el grado de severidad de impactos ambientales que esa actividad promueve y las diferentes perspectivas de desarrollo futuro en las distintas mesorregiones del estado. Las investigaciones se realizaron a través de la recolección de datos secundarios proporcionados por el Instituto de Protección Ambiental del Amazonas (IPAAM), que posee 1.382 pisciculturas registradas en su base de datos. Esta información fue organizada por microrregión (centro, suroeste, norte y sur) del estado, explorando sus principales indicadores técnicos, las infraestructuras de producción de formas jóvenes, el tamaño de los emprendimientos y el encuadramiento del grado de severidad de impacto ambiental por porte de la piscicultura . Los resultados obtenidos muestran que la piscicultura se practica mayoritariamente para el engorde de tambaqui con fines de comercialización, realizada en estructuras de tierra firme (viveros excavados y represas) en pequeñas áreas inundadas, con aplicación de sistema semi-intensivo de producción, siendo desarrollada cerca de (Manaus), teniendo como causa de su concentración factores logísticos y de infraestructura disponibles, con la mayoría de los emprendimientos encuadrados como bajo grado de severidad de impacto ambiental.

Palabras ClaVe: Colossoma macropomum, Amazonia, legislación ambiental, creación de peces.

\section{INTRODUÇÃO}

A valorização dada pela importância dos produtos pesqueiros, ocasionada tanto pelas suas qualidades nutricionais, refletida pela crescente demanda, estimulou o crescimento da piscicultura, tornando o desenvolvimento desta atividade uma tendência mundial (1). Apesar de condições propícias, a produção brasileira da piscicultura fica muito aquém do seu potencial (2).

Dados recentes da FAO mostram que o Brasil ocupa atualmente a $13^{\text {a }}$ posição em produção mundial da aquicultura, atrás de grandes produtores como China, Indonésia e Índia (1). Tal condição se deve ao fato da atividade se encontra pouco estruturada no país, sendo predominantemente informal e caracterizada pelo uso de métodos de criação artesanais (2)

De todas as regiões brasileiras, a Amazônia apresenta as condições ambientais mais favoráveis ao incremento da produção piscícola (3), sobretudo com a implantação de Parques aquícolas em reservatórios de águas públicas da União (4). No Brasil, o Amazonas possui grande potencial para expansão e sucesso da atividade no mercado nacional, visto que apresenta fatores propícios, tais como clima tropical, disponibilidade hídrica, topografia apropriada e variedade de espécies com valor de mercado e desempenho para a criação (5).

O setor aquícola no Brasil passou por um longo período em que as informações sobre a atividade foram ignoradas, sendo que não existiam na maioria dos estados brasileiros sistemas confiáveis de coleta de dados da produção da atividade (6). A disponibilidade de informações técnicas e científicas sobre comunidades produtoras de pescado tem como objetivo aprimorar seu conhecimento através de identificação de pontos 
deficientes e falhas estruturais, visando ampliar a produção para atender ao mercado, assegurando seu desenvolvimento tecnológico $(7,8)$.

Portanto, o objetivo deste trabalho foi descrever as características regionais da piscicultura praticada no estado do Amazonas e identificar, por mesorregião do estado, os principais indicadores técnicos dos cultivos, as infraestruturas de produção de formas jovens, o tamanho dos empreendimentos e o enquadramento do grau de severidade relativo ao impacto ambiental por porte da atividade de piscicultura, visando contribuir para o desenvolvimento desta atividade no Estado do Amazonas.

\section{METODOLOGIA}

O presente estudo foi realizado no estado do Amazonas, Brasil, a partir das informações de processos técnicos de pisciculturas cadastrados junto ao banco de dados do Instituto de Proteção Ambiental do Amazonas IPAAM, autarquia responsável pelo controle e regularização ambiental de atividades poluidoras no estado, que concedeu formalmente os dados para as investigaçóes.

As informações cedidas compuseram dados secundários da pesquisa, com um total de 1.382 empreendimentos formalizados até março de 2017. Todos os processos apresentaram características técnicas dos empreendimentos, mapas de localização, projetos executivos e memoriais descritivos, contendo dados relacionados a índices zootécnicos e indicadores produtivos, que foram utilizados neste trabalho para produção de cenários e panoramas atualizados da atividade piscícola no estado.

Além dos dados mencionados, também foram retiradas das informações cedidas pelo IPAAM os principais indicadores técnicos característicos (nível tecnológico de produção, potencial produtivo do setor, principais espécies trabalhadas) e outras informações consideradas úteis para o estudo (9).

Os dados foram tabulados em planilhas do Microsoft Office Excel 2016, para os posteriores tratamentos estatísticos e subsequentes interpretações dos resultados obtidos. A partir da análise dos dados foram calculadas a distribuição de frequência (simples absoluta), conforme sua localização geográfica dentro das subdivisões regionais (7) de arranjos (zonas fisiográficas) por mesorregiões (10) (Figura 1).

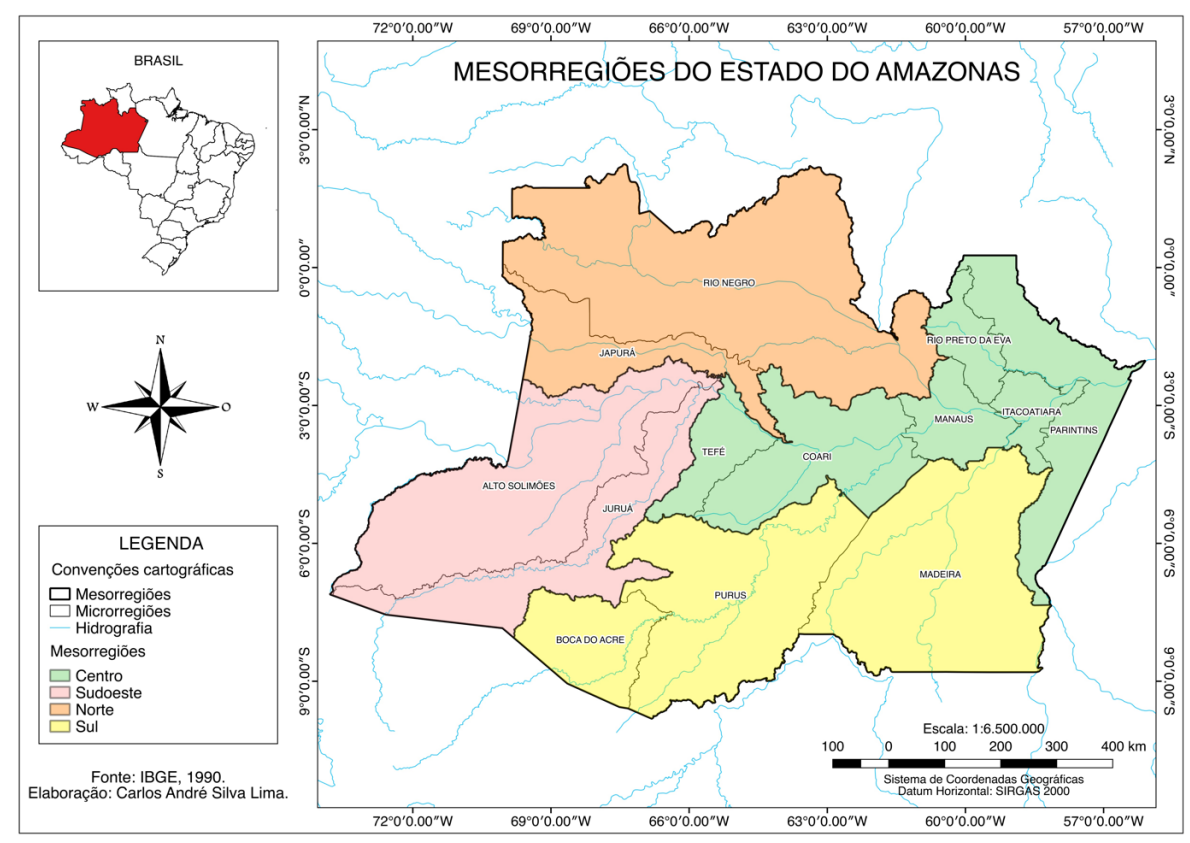

FIGURA 1

Mapa das mesorregiões do estado do Amazonas, Brasil IBGE, 1990. 


\section{RESULTADOS}

Com base nos dados coletados, foi verificado que a aquicultura no Amazonas é uma atividade desenvolvida em empreendimentos localizados em ambientes de terra firme (93,33\%) (Figura 2), compostos por viveiros escavados e barragens, os quais são destinados à piscicultura (99,49\%), com o uso de várias espécies (57,02\%) nativas, predominando, no entanto, o uso do tambaqui (92,40\%).

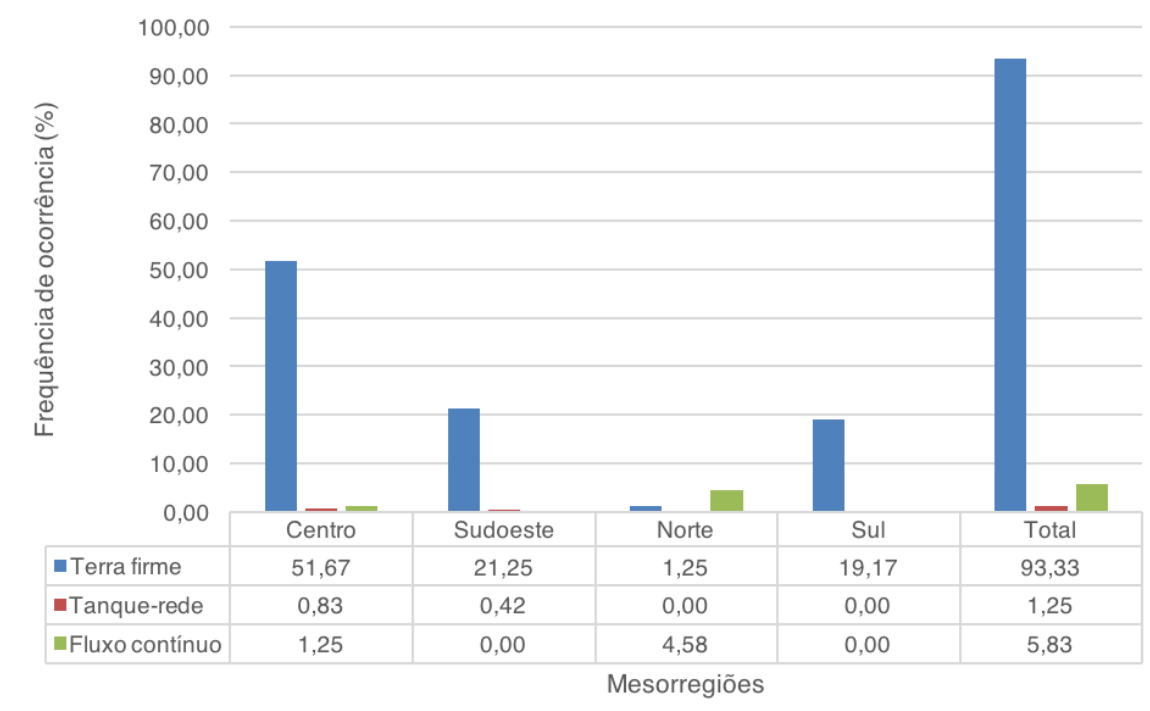

FIGURA 2

Frequência de ocorrência dos empreendimentos de piscicultura por tipo de estrutura de criação no estado do Amazonas, por mesorregiões e absoluto

IPAAM, 2017

O sistema semi-intensivo de produção (90,45\%) é utilizado estrategicamente no Amazonas, destinado preferencialmente a engorda (98,48\%). Apesar disso, identificou-se a ocorrência de empreendimentos (17) que praticam alevinagem e recria (1,95\%) no Amazonas, dedicando esforço no abastecimento de "sementes" para a atividade no estado, o que corresponde a uma capacidade superior a 25 milhóes de alevinos/ano (Tabela $1)$. 
TABELA 1

Distribuição de empreendimentos de piscicultura para a produção de alevinos no estado do Amazonas, por mesorregióes e municípios.

\begin{tabular}{|c|c|c|c|c|}
\hline Mesorregião $^{1}$ & Município $^{1}$ & Espécies criadas & Qtd. $^{2}$ & $\begin{array}{c}\text { Tipo de } \\
\text { empreendimento }\end{array}$ \\
\hline \multirow{14}{*}{ Centro } & Iranduba & Tambaqui & 1,0 & Particular \\
\hline & Iranduba & Tambaqui/Matrinxã & 1,0 & Particular \\
\hline & Itacoatiara & Tambaqui & 1,0 & Particular \\
\hline & Itacoatiara & Tambaqui & 1,5 & Particular \\
\hline & Itacoatiara & Tambaqui & 1,0 & Particular \\
\hline & Itacoatiara & Tambaqui & 1,5 & Particular \\
\hline & Manacapuru & Tambaqui & 2,0 & Particular \\
\hline & Manacapuru & Pirarucu & 0,5 & Particular \\
\hline & Manacapuru & Tambaqui/Matrinxã/Pirarucu & 2,0 & Particular \\
\hline & $\begin{array}{l}\text { Presidente } \\
\text { Figueiredo }\end{array}$ & Tambaqui/Matrinxẫ/Pirarucu & 1,5 & Particular \\
\hline & $\begin{array}{l}\text { Presidente } \\
\text { Figueiredo }\end{array}$ & Tambaqui/Matrinxã & 2,5 & Particular \\
\hline & $\begin{array}{l}\text { Presidente } \\
\text { Figueiredo }\end{array}$ & Tambaqui/Matrinxã & 3,5 & Pública \\
\hline & $\begin{array}{l}\text { Rio Preto da } \\
\text { Eva }\end{array}$ & Tambaqui/Matrinxã /Pirarucu & 1,5 & Particular \\
\hline & $\begin{array}{l}\text { Rio Preto da } \\
\text { Eva }\end{array}$ & Tambaqui/Matrinxã & 1,0 & Particular \\
\hline \multirow{2}{*}{ Sudoeste } & $\begin{array}{l}\text { Benjamin } \\
\text { Constant }\end{array}$ & Tambaqui/Matrinxã/Curimatã & 1,5 & Particular \\
\hline & $\begin{array}{l}\text { Nova Olinda } \\
\text { do Norte }\end{array}$ & Pirarucu & 0,5 & Particular \\
\hline Norte & - & - & 0,0 & - \\
\hline \multirow[t]{2}{*}{ Sul } & Humaitá & Tambaqui/Matrinxã/Pirarucu/Surubim & 1,5 & Pública \\
\hline & & Total & 25,0 & \\
\hline
\end{tabular}

Banco de dados do IPAAM, 2017

1 IBGE, 1990

2 Milhões de alevinos por iniciativa de produção ou lote

A principal finalidade das pisciculturas, incluindo-se aquelas que praticam engorda e alevinagem, segundo os resultados, é a comercialização (98,26\%), sendo pouco expressivo índice de produtores que desenvolvem a atividade com fins de subsistência $(7,38 \%)$.

Conforme critérios de classificação estabelecidos na legislação vigente do Conselho Nacional do Meio Ambiente, Resolução n ${ }^{\circ} 413$ de 26 de julho de 2009, a piscicultura no Amazonas se destaca como atividade composta por empreendimentos de pequeno porte (93,78\%), mais concentrada geograficamente na mesorregião Centro (83,07\%), e com maior agrupamento de piscicultores na microrregião de Manaus $(40,59 \%)$ (Tabela 2). 
TABELA 2

Quantidade, área alagada/útil e distribuição geográfica dos empreendimentos piscícolas do estado do Amazonas

\begin{tabular}{|c|c|c|c|c|c|c|c|c|c|}
\hline & & & & & & Área alag & ada/út & & \\
\hline Divis & Regional $^{1}$ & Pisci & culturas & $\begin{array}{r}\text { Terra } \\
\text { (hect }\end{array}$ & $\begin{array}{l}\text { firme } \\
\text { are) }\end{array}$ & $\begin{array}{r}\text { Tanque } \\
\left(\mathrm{m}^{3}\right.\end{array}$ & -rede & $\begin{array}{r}\text { Fluxo co } \\
(\mathrm{m}\end{array}$ & tínuo \\
\hline Mesorreg & ${ }^{1}$ Microrregião & Qtd. & (\%) & Qtd. & (\%) & Qtd. & (\%) & Qtd. & (\%) \\
\hline & Coari & 149 & 10,78 & 150,30 & 5,72 & 0,00 & 0,00 & 0,00 & 0,00 \\
\hline & Itacoatiara & 92 & 6,66 & 304,90 & 11,60 & $8.502,40$ & 48,02 & $4.591,20$ & 21,45 \\
\hline & Manaus & 561 & 40,59 & $1.081,76$ & 41,16 & $3.166,51$ & 17,88 & $8.158,81$ & 38,11 \\
\hline Centro & Parintins & 49 & 3,55 & 57,81 & 2,20 & $4.090,24$ & 23,10 & 0,00 & 0,00 \\
\hline & $\begin{array}{l}\text { Rio Preto da } \\
\text { Eva }\end{array}$ & 288 & 20,84 & 592,29 & 22,54 & 517,44 & 2,92 & $5.612,20$ & 26,21 \\
\hline & Tefé & 9 & 0,65 & 18,26 & 0,69 & 0,00 & 0,00 & 0,00 & 0,00 \\
\hline Sudoeste & Alto Solimões & 71 & 5,14 & 106,62 & 4,06 & 170,00 & 0,96 & 0,00 & 0,00 \\
\hline sudoeste & Juruá & 29 & 2,10 & 53,86 & 2,05 & 0,00 & 0,00 & 0,00 & 0,00 \\
\hline Norte & Japurá & 1 & 0,07 & 0,69 & 0,03 & 0,00 & 0,00 & 0,00 & 0,00 \\
\hline Norte & Rio Negro & 15 & 1,09 & 9,82 & 0,37 & 0,00 & 0,00 & $2.946,63$ & 13,76 \\
\hline & Boca do Acre & 12 & 0,87 & 18,67 & 0,71 & 0,00 & 0,00 & 0,00 & 0,00 \\
\hline Sul & Madeira & 97 & 7,02 & 194,00 & 7,38 & $1.260,00$ & 7,12 & 100,00 & 0,47 \\
\hline & Purus & 9 & 0,65 & 39,04 & 1,49 & 0,00 & 0,00 & 0,00 & 0,00 \\
\hline & otal & 1.382 & 100,00 & $2.628,02$ & 100,00 & $17.706,59$ & 100,00 & $21.408,8$ & 100,00 \\
\hline
\end{tabular}

Banco de dados do IPAAM, 2017

A produção estimada para o estado do Amazonas foi calculada em 20,77 mil toneladas de pescado para o período de aplicação deste estudo, oriundas, principalmente de estruturas disposta em terra firme (93,86\%). A produção estimada para este tipo de ambiente chega a 19,50 mil toneladas de pescado produzido, considerando a área total de espelho d'água (2.628,02 hectares) e quantidade de pisciculturas contabilizadas (1.289). Quanto à distribuição de produção por mesorregião, observa-se que a mesorregião Centro concentra $84,37 \%$ do total produzido no estado, com 17,52 mil toneladas acumuladas, sendo 16.367,27 mil toneladas em terra firme (Figura 3). 


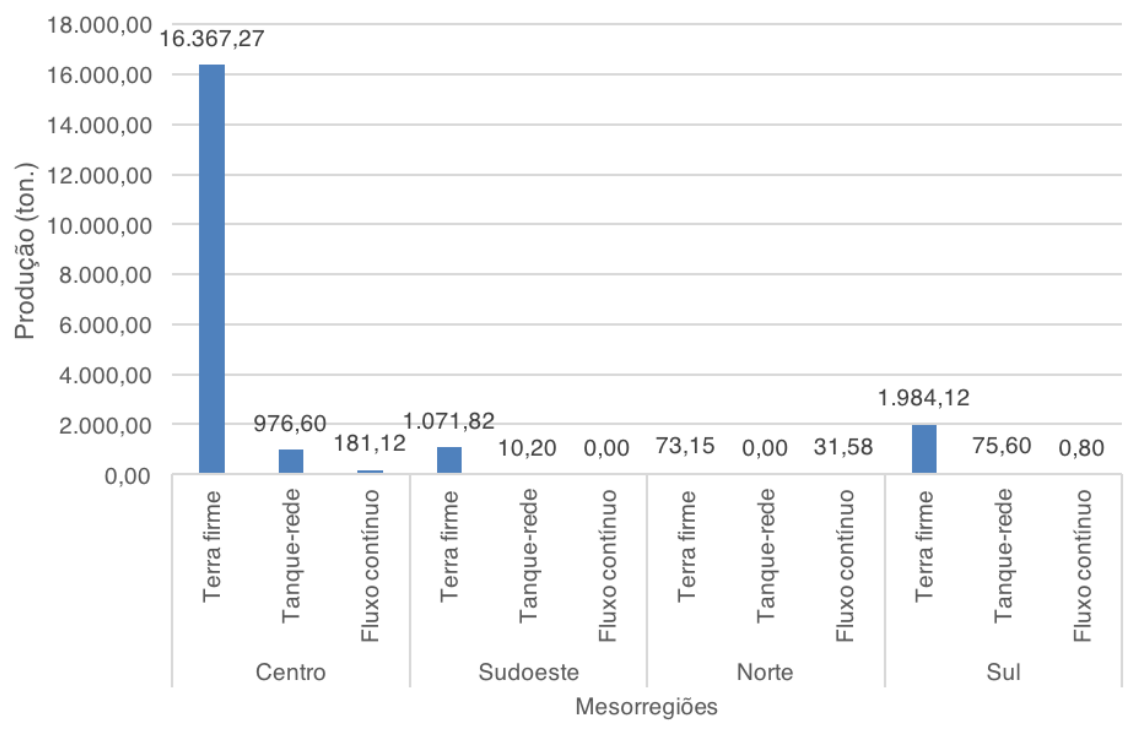

FIGURA 3

Frequência de ocorrência da produção de pescado por categoria de estrutura de criação e mesorregiões do estado do Amazonas.

IPAAM, 2017

Identificou-se, através dos critérios ambientais e de características das infraestruturas para classificação do potencial de severidade da atividade estabelecidos pela Resolução n. 413 de 2009, que de um total de 1.382 empreendimentos registrados junto à fonte de dados secundária, somente 46 (3,33\%) são classificados como possuidores de "significativo" potencial poluidor (Tabela 3). 
TABELA 3

Resultado da distribuição de empreendimentos de piscicultura do estado do Amazonas conforme enquadramento legal de potencial de severidade estabelecido na Resolução CONAMA n. 413 de 2009.

\begin{tabular}{lcccc}
\hline \multirow{2}{*}{ Porte $^{1}$} & \multicolumn{3}{c}{ Potencial de severidade $^{2}$} & \multirow{2}{*}{ Total } \\
\cline { 2 - 4 } & Baixo(B) & Médio(M) & Alto(A) & \\
\hline Pequeno(P) & $* 946(\mathrm{~PB})$ & $* 350(\mathrm{PM})$ & ${ }^{*} 0(\mathrm{PA})$ & 1.296 \\
Médio(M) & $* 40(\mathrm{MB})$ & $* * 44(\mathrm{MM})$ & $* * 0(\mathrm{MA})$ & 84 \\
Grande(G) & $* * 0(\mathrm{~GB})$ & $* * 2(\mathrm{GM})$ & ${ }^{*} 0(\mathrm{GA})$ & 2 \\
\hline \multicolumn{1}{c}{ Total geral } & 986 & 396 & 0 & 1.382 \\
\hline 1
\end{tabular}

1;2 Resolução CONAMA n. 413 de 2009.

Nota $^{1}:(\mathrm{P})=$ Terra firme: $<5,0$ hectares; Tanque-rede: $<1.000$

$\mathrm{m}^{3}$; Fluxo contínuo: $<1.000 \mathrm{~m}^{3}$. (M) = Terra firme: 5,0 a 50,0

hectares; Tanque-rede: 1.000 a $5.000 \mathrm{~m} 3$; Fluxo continuo: 1.000

a $5.000 \mathrm{~m}^{3}$. (G) = Terra firme: $>50,0$ hectares; Tanque-rede: $>$

$5.000 \mathrm{~m}^{3}$; Fluxo contínuo: $>5.000 \mathrm{~m}^{3}$.

Nota $^{2}$ : (B) = Sistema extensivo com espécie autóctone/nativa não-

carnivora; Sistema extensivo com espécie autóctone/nativa

carnivora; Sistema semi-intensivo com espécie autóctone/nativa

não-carnivora. $(M)$ = Sistema extensivo com espécie

alóctone /exótica não-carnivora; Sistema extensivo com espécie alóctone/exótica carnivora; Sistema semi-intensivo com espécie autóctone/nativa carnivora; Sistema semi-intensivo com espécie alóctone/exótica não-carnivora; Sistema intensivo com espécie autóctone/nativa não-carnivora; Sistema intensivo com espécie autóctone/nativa carnivora. (A) = Sistema semi-intensivo com espécie alóctone lexótica carnivora; Sistema intensivo com espécie alóctone jexótica não-carnivora; Sistema intensivo com espécie alóctone/exótica carnivora. *Potencial de severidade "nãosignificativo". "*Potencial de severidade "Significativo".

Banco de dados do IPAAM, 2017

Ao se verificar entre as mesorregiões do estado do Amazonas, identificou-se que aqueles empreendimentos com elevando ou "significativo" potencial de geração de danos ambientais (severidade), conforme legislação vigente, estão mais concentrados na mesorregião Centro (91,30\%), seguida das mesorregióes Sudoeste $(4,35 \%)$ e Sul (4,35\%) (Figura 4). 


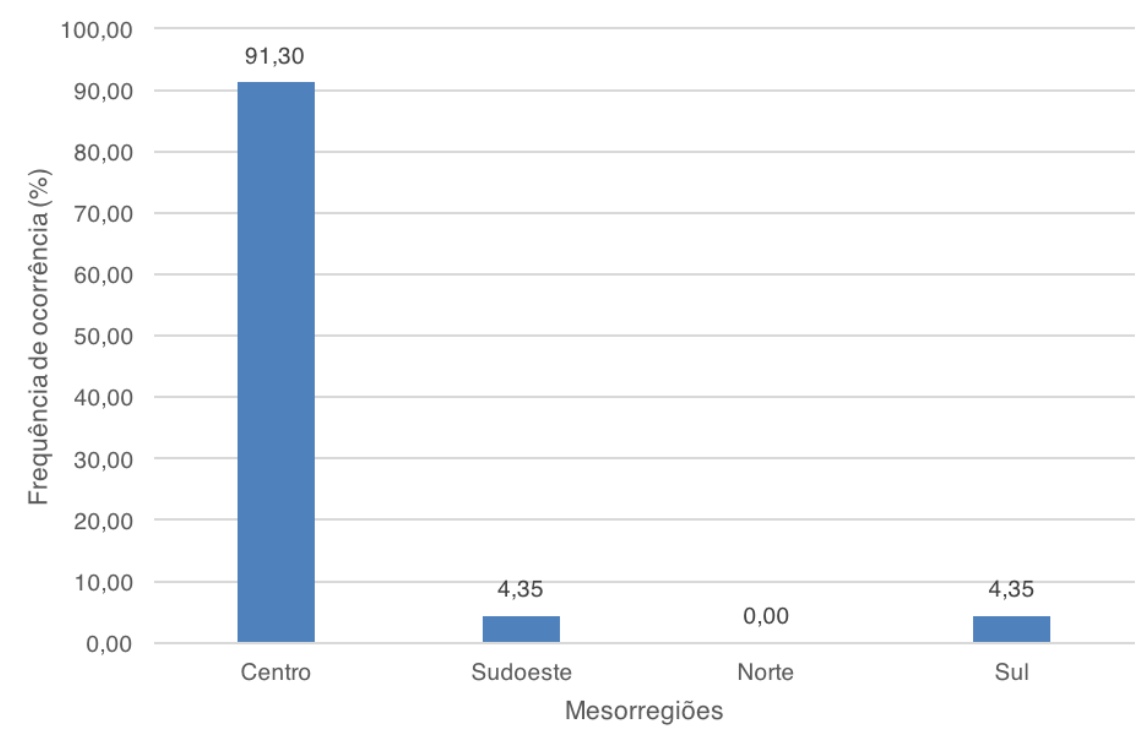

FIGURA 4

Frequência de ocorrência dos empreendimentos de piscicultura enquadrados como possuidores de significativo potencial de severidade ambiental, conforme Resolução CONAMA n. 413 de 2009, por mesorregiões do estado IPAAM, 2017

\section{DISCUSSÃO}

Comparativamente, constata-se que os valores obtidos para o estado do Amazonas neste estudo corroboram resultados levantados na literatura, ao informarem que a piscicultura desenvolvida em viveiros escavados e barragens se sobressaem como categoria de estrutura de terra firme mais utilizados no Brasil $(11,12)$.

Diversos estudos identificam que a aquicultura no Amazonas é formada predominantemente pela piscicultura com espécies nativas, sendo o tambaqui a principal espécie $(7,13,18)$. Apesar disto, pode ser identificada a existência de espécies exóticas como a tilápia (Oreochromis niloticus) sendo criadas no estado $(13,14,19)$.

Observa-se, no entanto, que existem restrições legais que condicionam à obrigatoriedade do uso de espécies nativas no Amazonas, em contraste às outras regiões do país (12, 20, 21, 22, 23), apresentando-se como fator principal o impedimento de utilização de espécies alóctones ou exóticas em consequência de instrumentos normativos restritivos (24).

Em relação ao sistema de produção, vários estudos verificam a predominância de sistemas semi-intensivos no Brasil (12) e no Amazonas (15) relacionados ao baixo nível de aplicação de tecnologia, como identificado neste estudo, bem como a escolha da engorda como objetivo principal do empreendimento (25), sendo a comercialização a finalidade proposta para a atividade (16).

Quanto à alevinagem, esta se comporta como um modelo de atividade piscícola que exige elevado conhecimento técnico e formação específica $(26,27)$, além de possuir complicado processo de controle do ambiente e demandar onerosa estrutura de produção e capital de giro para custeio $(27,28)$. No entanto, apesar de depender efetivamente do uso e difusão de tecnologia $(26,28)$, a alevinagem representa uma etapa importante e que apresenta grande potencial de retorno financeiro $(29,30)$, compondo o elo de maior relevância na cadeia produtiva pela imprescindibilidade em suprir a crescente demanda por pescado no processo de engorda $(2,27,30)$. 
Assim, ao se observarem as condições relacionadas à capacidade de oferta para o fornecimento de alevinos no Amazonas, pôde-se verificar que apesar do agrupamento dos empreendimentos na mesorregiáo com maior produtividade (Centro), existem atualmente suficiente infraestrutura para o fornecimento das principais espécies (à exceção do pirarucu) para atender de forma imediata à demanda dos piscicultores em todo o Estado (5), evitando assim, a dependência do fornecimento de alevinos de outras localidades (31).

Sobre a localização das pisciculturas e valores de produção, assim como apurado por Gandra (13) e corroborado posteriormente por Pantoja-Lima et al (15), a Região Metropolitana de Manaus (RMM) agrupa a maior quantidade de pisciculturas em seus territórios, podendo chegar a $85 \%$ do total existente no estado devido a fatores estruturais (logística) e proximidade do mercado consumidor (Manaus) para aquisição de insumos com menores custos e facilidade na comercialização do pescado $(13,32)$, sendo esta característica verificada nos resultados apresentados.

Embora a região metropolitana de Manaus apresente todas as características propícias para a piscicultura, a maioria dos empreendimentos é de porte pequeno, com pouca aplicação tecnológica no sistema de criação e com uso de espécies que não trazem grandes riscos ambientais em decorrência de suas características ecológicas, fatores estes que fazem com que a atividade apresente bons índices de avaliação quanto ao ordenamento e controle de impactos sobre a sua produção.

Tais particularidades fazem como estas sejam classificadas como de baixo grau de severidade ambiental, de acordo com a legislação ambiental brasileira vigente, similar ao que ocorre na maioria dos empreendimentos de outras regiões da Amazônia (5, 20, 30, 31, 32). Esta avaliação da sustentabilidade ambiental da atividade da piscicultura, pautada em assuntos de ordem legal, através de marcos administrativos e jurídicos idôneos, garantem a aplicação e prática da atividade aquícola de forma ambientalmente sustentável (33).

Em conclusão a piscicultura no estado do Amazonas atualmente vem sendo praticada com fins comerciais, majoritariamente em empreendimentos de terra firme em pequenas áreas alagadas, onde a produção é priorizada para a espécie tambaqui com objetivo de engorda em sistemas semi-intensivos de criação.

No entanto, um perfil de heterogeneidade é identificado quanto às particularidades dos sistemas de produção, porte do empreendimento, bem como a localização geográfica, os quais podem ser atribuídos às influências relacionadas às peculiaridades geográficas, sociais e econômicas regionais específicas, tais como, a concentração do mercado consumidor de destinação do pescado produzido localizado na cidade de Manaus, o que contribui para a aglomeração da sua prática no entorno e em áreas adjacentes, que possuem boa infraestrutura logística para abastecimento de insumos e escoamento da produção no provimento da demanda do mercado local. Essa piscicultura praticada no Amazonas é realizada em sua maioria em propriedades de pequeno porte e estão enquadradas com baixo grau de severidade e impacto ambiental.

\section{AGRADECIMENTOS}

Os autores agradecem ao Instituto de Proteção Ambiental do Amazonas - IPAAM, pelo apoio na disponibilização dos dados. Ao programa de PPG-CIPET pela oportunidade de realizar o presente estudo. À Fundação de Amparo à Pesquisa no Estado do Amazonas, por concessão de bolsa ao orientador.

\section{REFERÊNCIAS}

1. FAO. The State of World Fisheries and Aquaculture 2018 - Meeting the sustainable development goals. Rome: FAO; 2018. URL Available in: http://www.fao.org/state-of-fisheries-aquaculture/en/

2. Sidônio L, Cavalcanti I, Capanema L, Morch R, Magalhães G, Lima J. et al. Panorama da aquicultura no Brasil: desafios e oportunidades. Agroindústria, BNDES Setorial. 2012; 35:421-463. URL Available in: https://www.bndes.gov.br/SiteBNDES/export/sites/default/bndes_pt/Galerias/Arquivos/conhecimento/ bnset/set3512.pdf

3. MPA - Ministério da Pesca e Aquicultura. Amazônia, Aquicultura e Pesca - Plano de Desenvolvimento Sustentável. Brasília: MPA; 2009. URL Available 
in: https://www2.camara.leg.br/atividade-legislativa/comissoes/comissoes-permanentes/cindra/audienciaspublicas/audiencias/audiencias-publicas-maio/20-05-2009-audiencia-publica-plano-amazonia-sustentavel-deaquicultura-e-pesca/PASAP\%2017_04_09\%20versao\%201\%201.pdf/view

4. Bueno GW, Ostrensky A, Canzi C, Matos FT, Roubach R. Implementation of aquaculture parks in Federal Government waters in Brazil. Aquaculture. 2015; 7(1):1-12. DOI: https://doi.org/10.1111/raq.12045

5. Lopes MLB, Costa PA, Santos JSB, Cunha SJT, Santos MAS, Santana AC. Mercado e dinâmica espacial da cadeia produtiva da pesca e aquicultura na Amazônia. Banco da Amazônia: Estudos setoriais 7. Belém: BASA, 2010. URL Available in: https://www.yumpu.com/pt/document/read/14699069/estudo-banco-da-amazonia/6

6. Kubitza F, Campos JL, Ono EA, Istichuk PI. Panorama da Piscicultura no Brasil: Estatísticas, espécies, polos de produção e fatores limitantes à expansão da atividade. Panorama da Aquicultura. 2012; 22(132):14-23. URL Available in: https://panoramadaaquicultura.com.br/edicoes/edicao-132/

7. Oliveira AM, Val VMFA, Val AL. Caracterização da atividade de piscicultura nas mesorregiões do estado do Amazonas, Amazônia brasileira. Rev Colomb Cienc Anim. 2012; 4(1):154-162. DOI: https:// doi.org/10.24188/recia.v4.n1.2012.303

8. Rocha CMC, Resende EK, Routledge EAB, Lundstedt LM. Avanc\#os na pesquisa e no desenvolvimento da aquicultura brasileira. Pesq Agropec Bras. 2013; 48(8):4-6. DOI: https://doi.10.1590/ S0100-204X2013000800iii

9. Silva NA, Lima EBNR, Silvino ANO, Santos AA, Silva JB; Lima JB. Caracterização espacial das pisciculturas na Bacia do Rio Cuiabá/MT. Engenharia Ambiental - Espírito Santo do Pinhal. 2008; 5(3):47-62. URL Available in: http://ferramentas.unipinhal.edu.br/engenhariaambiental/viewarticle.php?id=139

10. IBGE - Instituto Brasileiro de Geografia e Estatística. Divisão Regional do Brasil em Mesorregióes e Microrregiões Geográficas. IBGE: Rio de Janeiro, Brasil: 1990. https://biblioteca.ibge.gov.br/index.php/biblioteca-catalogo? view $=$ detalhes\&id $=22269$

11. Pedroza FMX, Rodrigues APO, Rezende FP. Dinâmica da produção de tambaqui e demais peixes redondos no Brasil. Embrapa Pesca e Aquicultura: Brasil; 2016. URL Available in: https://www.embrapa.br/pesca-e-aquicultura/busca-de-publicacoes/-/publicacao/1041302/dinamica-daproducao-de-tambaqui-e-demais-peixes-redondos-no-brasil

12. Zacardi DM, Lima, MAS, Nascimento MM, Zanetti CRM. Caracterização socioeconômica e produtiva da aquicultura desenvolvida em Santarém, Pará. Acta Fish. 2017; 5(3):102-112. DOI: http://dx.doi.org/10.2312/ Actafish.2017.5.3.102-112

13. Gandra AL. O Mercado do Pescado da Região Metropolitana de Manaus. Infopesca, FAO: Brasil; 2010. URL Available in: http://www.infopesca.org/sites/default/files/complemento/publilibreacceso/282/Manauscompleto.pdf

14. Nakauth ACSS, Nakauth RF, Nóvoa NACB. Características da piscicultura no município de Tabatinga-AM. Rev Igapó. 2015; 9(2):54-64. URL Availabre in: http://200.129.168.183/ojs/index.php/igapo/article/view/311

15. Pantoja-Lima J, Santos SM, Oliveira AT, Araújo RL, Silva-Jr JAL. Aride PHR. Pró-rural aquicultura: relatos das principais ações de extensão tecnológica e um panorama do setor aquícola do estado do Amazonas, Brasil. Nexus Revista de Extensão do IFAM. 2015; 1(1):35-45. URL Available in: http://200.129.168.183/ojs_proex/ index.php/Nexus/article/view/6

16. Estevão-Rodrigues TT. Pantoja-Lima J. Piscicultura familiar, assistência técnica e práticas de manejo colheita e pós colheita: estudo de caso da região metropolitana de Manaus - Amazonas, Brasil. Revista Observatorio de la Economía Latinoamericana, Brasil. 2017. URL Availabre In: http://www.eumed.net/cursecon/ecolat/br/17/ piscicultura.html

17. Campos JL, Ono EA, Istchuk PI. A Cadeia de Produção e o Preço do Tambaqui. Panorama da Aquicultura. 2015; 25(149):42-45. URL Available in: https://www.novaaqua.com.br/site/artigos/Pan149_Tambaqui.pdf

18. Pantoja-Lima J, Santos SM, Oliveira AT, Araujo RL, Silva Junior JAL, Bernardino G, et al. Pesquisa e transferência de tecnologia aliadas para desenvolvimento da aquicultura no Estado do Amazonas. Em: Aquicultura no 
Brasil: novas perspectivas. [Vol. 2]. São Carlos: Pedro \& João; 2015. URL Available in: https://pt.scribd.com/ document/371848006/Livro-Pronto-volume-II-Aquicultura-No-Brasil

19. Martins-Jr H. Caracterização da piscicultura do tambaqui (Colossoma macropomum) e dos seus efluentes na despesca: Subsídios para a implementação das boas práticas de manejo da piscicultura no município de Rio Preto da Eva/AM. [Tese doutorado]. Manaus, Amazonas, Brasil: Instituto Nacional de Pesquisas da Amazônia; 2009. URL Available in: http://bdtd.inpa.gov.br/handle/tede/1487

20. Leonel APS. Viabilidade econômica de produtos a\# base de tilápia para alimentação escolar nos municípios de Toledo-PR e Marechal Cândido Rondon-PR. [Tese doutorado]. Universidade Estadual Paulista, Centro de Aquicultura da UNESP: Jaboticabal, Brasil; 2016. URL Available in: http://hdl.handle.net/11449/148648

21. Mendes AI, Carvalho MC. Caracterização da piscicultura em tanques-rede no município de Rubinéia-SP: um estudo de caso. Rev do Agronegócio - Reagro, Jales, 2016; 5(1):16-33. URL Available in: http://www.fatecjales.edu.br/reagro/images/artigos/1a_edicao/volume5/caracterizacao-dapiscicultura-em-tanques-rede.pdf

22. Ribeiro-Neto TF, Silva AHG, Guimarães I, Gomes MVT. Piscicultura familiar extensiva no baixo São Francisco, estado de Sergipe, Brasil. Acta Fish Aquat Res. 2016; 4(1):62-69. DOI: http://dx.doi.org/10.2312/ Actafish.2016.4.1.62-69

23. Silva FNL, Costa MSM, Malcher CS, Medeiros LR, Macedo ARG, Freitas, HLC, Souza RAL. Cultivo de organismos aquáticos: uma proposta de desenvolvimento rural na Ilha de João Pilatos, Ananindeua - Pará - Brasil. Pubvet. 2017; 11(5):424-432. DOI: https://doi.org/10.22256/pubvet.v11n5.424-432

24. Lei $\mathrm{n}^{\circ} 3.802$, de 29 de agosto de 2012. Disciplina a atividade de aquicultura no Estado do Amazonas e dá outras providências. Diário Oficial do Estado do Amazonas: Manaus, Brasil; 2012. URL Available in: https:// www.legisweb.com.br/legislacao/?id=244797

25. Dotti A, Valejo PAP, Russo MR. Licenciamento ambiental na piscicultura com enfoque na pequena propriedade: uma ferramenta de gestão ambiental. Revista Ibero\#Americana de Ciências Ambientais, Aquidabã. 2012; 3(1):6\#16. DOI: https://doi.org/10.6008/ESS2179-6858.2012.001.0001

26. Hermes CA. Sistema agroindustrial da tilápia na região de Toledo - PR e comportamento de custos e receitas. [Tese doutorado]. Universidade Estadual Paulista, Centro de Aquicultura da UNESP - CAUNESP. Jaboticabal: Brasil, 2009. URL Available in: http://hdl.handle.net/11449/100243

27. Brabo MF, Reis MHD, Veras GC, Silva MJM, Souza ASL, Souza RAL. Viabilidade econômica da produção de alevinos de espécies reofílicas em uma piscicultura na Amazônia Oriental. Bol Inst Pesca São Paulo. 2015; 41(3):677-685. URL Available in: https://www.pesca.sp.gov.br/41_3_677-685.pdf

28. Sanches EG, Tosta GAM, Souza-Filho JJ. Viabilidade econômica da produção de formas jovens de bijupira\# (Rachycentron canadum). Bol Inst Pesca, São Paulo, 2013; 39(1):15-26. URL Available in: https:// www.pesca.sp.gov.br/39_1_15-26.pdf

29. Andrade DR, Shigueki YG. O manejo da reprodução natural e artificial e sua importância na produção de peixes no Brasil. Rev Bras de Reprod Anim. 2003; 27(2):166-172. URL Available in: https://www.researchgate.net/ publication/237659067_O_MANEJO_DA_REPRODUCAO_NATURAL_E_ARTIFICIAL_E_SUA_IMPORTANCI

30. Lopes MC, Silva NJR, Henrique MB. Custos e viabilidade econômica da produção de alevinos de lambaris reproduzidos artificialmente. Informações Econômicas, 2014; 44(6):60-68. URL Available in: http:// www.iea.sp.gov.br/ftpiea/publicacoes/ie/2014/tec6-1214.pdf

31. Oliveira ASC, Souza RAL, Melo NFA. Estado da Arte da Piscicultura na Mesorregião Sudoeste Paraense Amazônia Oriental. Bol Téc Cient Cepnor. 2014; 14(1):33-38. DOI: https://doi.org/10.17080/1676-5664/ btcc.v14n1p33-38

32. Brabo MF, Veras GC, Campelo DAV, Costa JWP, Rabelo LP. Piscicultura no estado do Para\#: custo de produção e indicadores econômicos. $1^{\text {a }}$. ed. UFP: Cartilha. Bragança: 2016. URL Available in: http://www.institutovitoriaregia.org.br/site/admin/arquivo/lhe515_Cartilha\%20-\%20Custo \%20de\%20producao\%20e\%20indicadores\%20economicos.pdf 
33. Borges AF, Borges MAC, Rezende JLP, Durigon MSGF, Corte AR, Vieira, FAB, et al. Desempenho Ambiental da Piscicultura na Amazônia Ocidental Brasileira. Gl Sci Technol, Rio Verde, 2013; 6(1):141-152. DOI: https:// doi.org/10.14688/1984-3801.v06n01a13

Los autores permiten a RECIA reimprimir el material publicado en él. En caso de que un autor quiera traducir o usar una publicación parcial o completa de nuestro Diario, el autor debe obtener un permiso por escrito del editor de la revista.

CC BY 